\title{
DIVERSIDAD Y ENDEMISMO DE LOS ESCARABAJOS (INSECTA: COLEOPTERA) EN LA HISPANIOLA, ANTILLAS MAYORES
}

\author{
Daniel E. Perez-Gelabert
}

\begin{abstract}
Department of Entomology, U.S. National Museum of Natural History, Smithsonian Institution, P.O. Box 37012, Washington, DC, 20013-7012, USA. perezd@si.edu
\end{abstract}

\section{RESUMEN}

Se analizaron parámetros cuantitativos sobre la diversidad de escarabajos en La Hispaniola y se la comparó con la coleopterofauna de otras islas del Caribe. Se encontró que para La Hispaniola se han citado 1,973 especies actuales, distribuidas en 75 familias, y 184 especies fósiles, correspondientes a 29 familias. Las tres familias con mayor número de especies son Curculionidae (253), Chrysomelidae (211) y Staphylinidae (206). Más de la mitad de las especies $(1,045$ o $53.0 \%)$ y 28 géneros son endémicos de la isla. Se identificaron 56 especies como introducidas. A pesar de importantes avances en la caracterización de esta fauna en los últimos 40 años, puede decirse que los escarabajos de La Hispaniola se conocen aún de forma preliminar. Se estima que la fauna total de escarabajos en la isla contendría entre 3,200 - 4,000 especies. Esto refuerza la designación de las islas del Caribe como uno de los "hotspots" de la biodiversidad mundial. Toda esta diversidad biológica está amenazada por la rápida transformación y destrucción de los hábitats naturales en la isla.

Palabras clave: insectos, Coleoptera, fauna, República Dominicana, Haití, La Hispaniola, Antillas.

\section{ABSTRACT}

Quantitative parameters on the diversity of beetles in Hispaniola were analyzed and this diversity was compared to the coleopterofauna of other Caribbean islands. It was found that 1,973 recent species of beetles in 75 families and 184 fossil species in 29 families have been recorded for Hispaniola. The three families with the largest numbers of species are Curculionidae (253), Chrysomelidae (211) and Staphylinidae (206). More than half of the species (1,045 or $53.0 \%$ ) and 28 genera are endemic to the island. Fifty-six species were identified as introduced. Despite important advances in the characterization of this fauna made during the last 40 years, it can be affirmed that knowledge on Hispaniolan beetles is still preliminary. It is estimated that the total fauna of beetles in Hispaniola would contain between 3,200 - 4,000 species. All this biological diversity is threatened by the rapid transformation and destruction of the natural habitats in the island. Thus this reinforces the designation of the Caribbean islands as one of the world's "hotspots" of biodiversity.

Key words: insects, Coleoptera, fauna, Dominican Republic, Haiti, Hispaniola, Antilles.

\section{INTRODUCCIÓN}

Los escarabajos son insectos que se caracterizan por poseer un par de alas endurecidas (élitros) que protegen un segundo par de alas membranosas utilizadas para el vuelo. Conjuntamente, las alas membranosas y los élitros recubren el abdomen produciendo un cuerpo compacto que puede escurrirse fácilmente entre escondrijos. Pero los escarabajos tienen una morfología muy variable, habiendo muchos braquípteros o completamente ápteros. Su amplia variedad de formas y tamaños se relaciona con la gran diversidad de hábitats y nichos ecológicos que ocupan. Otra adaptación que pudiera contribuir a su extraordinaria diversidad es su metamorfosis completa, 
implicando esto la existencia de larvas y adultos que explotan nichos ecológicos distintos. Los escarabajos ocupan casi todos los hábitats, exceptuando los océanos. Se han descrito entre 360,000 - 400,000 especies de escarabajos y cerca de un millón de especies de insectos (Chapman, 2009), por lo que la diversidad de escarabajos, el grupo biológico de mayor diversidad en el mundo, representaría entre $36-40 \%$ de todos los insectos. Los escarabajos explotan todas las fuentes posibles de alimento, por lo que incluyen especies fitófagas, micófagas, saprófagas, coprófagas, depredadoras, parásitas y parasitoides. Muchas especies son plagas importantes de las plantas cultivadas, de la madera y de productos almacenados. Otras especies son beneficiosas por su contribución como polinizadores, recicladores de materia orgánica y por su papel como depredadores de otros insectos (control biológico).

La Hispaniola $\left(76,480 \mathrm{~km}^{2}\right.$ ) es la segunda isla en extensión territorial de las Antillas Mayores, ocupando la República Dominicana sus dos tercios orientales y Haití el tercio occidental. Esta isla se caracteriza por la variabilidad de su relieve topográfico que incluye una alta proporción de paisajes montañosos distribuidos en cinco cordilleras y las montañas más altas en toda la región de las Antillas. La interacción de los vientos alisios con sus montañas influye en la cantidad y en los patrones de lluvia. Esto produce una marcada variabilidad de la humedad y temperaturas a diferentes niveles altitudinales que, combinado con la variedad de suelos, se traduce en un mosaico de microclimas, ambientes y bosques (bosques secos, bosques latifoliados, bosques de coníferas y bosques nublados) por toda la isla.

Las Antillas Mayores se originan como parte de un arco insular Cretácico que fue trasladado por tectonismo de la placa del Caribe. La porción Norte de La Hispaniola estuvo conectada con Cuba y Puerto Rico durante el Oligoceno y el Mioceno, posteriormente, esas conexiones terrestres desaparecieron a causa del tectonismo. La Hispaniola actual es el producto de dos paleoislas principales previamente independientes, las que finalmente se unieron a mediados del Mioceno (Pindell \& Barrett, 1990). La evolución de la fauna actual en las Antillas ha sido moldeada por la compleja historia geológica de la región del Caribe. Para explicar su origen se han propuesto dos modelos alternativos, vicarianza (e. g., Rosen, 1976) y dispersalismo (e. g., Hedges, 1996). La vicarianza ocurriría cuando, luego de la desaparición de conexiones terrestres con el continente, poblaciones de especies que antes tenían una amplia distribución quedaran aisladas, divergiendo subsecuentemente de sus ancestros. En el dispersalismo, las especies poblarían las islas a través del mar (en balsas de vegetación) y por el aire. El origen de la fauna caribeña probablemente incluya ambos fenómenos, aunque se debate cual mecanismo habría sido predominante. Las afinidades de la biota de La Hispaniola y las Antillas son con América Central y el norte de América del Sur.

La biota actual de La Hispaniola posee características insulares propias, estando ausentes muchos grupos de animales y plantas típicos del continente. Pero la gran mayoría de los órdenes de hexápodos están representados en la fauna actual. Los Notoptera (Grylloblattodea y Mantophasmatodea) y los Mecoptera no se encuentran en las islas del Caribe. Los órdenes Archaeognatha (=Microcoryphia) y Diplura todavía no se han reportado de La Hispaniola, pero deben estar presentes. Megaloptera y Plecoptera, sólo se han encontrado como fósiles en el ámbar dominicano, por lo que parecen haberse extinguido en la isla. En términos botánicos, la flora y bosques de La Hispaniola pueden considerarse diversos. La flora incluye aproximadamente 6,000 especies de plantas vasculares en 181 familias, siendo endémicas más de un tercio de estas (Mejía y García, 2007). La estrecha asociación de muchos artrópodos con la vegetación, la complejidad de los bosques y los nichos que estos crean, son determinantes importantes en la diversificación evolutiva de los insectos. Por esto, la destrucción de hábitats es probablemente el factor más importante que afecta la conservación de las poblaciones de insectos. Las severas 
transformaciones que han experimentado los bosques en el territorio haitiano, y muchas áreas del territorio dominicano, han debido producir múltiples extinciones locales. En el presente trabajo se examinan algunos aspectos sobre la diversidad y el endemismo de los escarabajos en La Hispaniola, como grupo y por familias.

\section{OBJETIVO}

- Analizar parámetros cuantitativos sobre la diversidad de escarabajos en La Hispaniola y compararlos con los correspondientes a otras islas del Caribe.

\section{MATERIALES Y MÉTODOS}

La diversidad de escarabajos actuales y fósiles citados de La Hispaniola fue inventariada en su conjunto, por primera vez, por Perez-Gelabert (2008). Para el presente trabajo se actualizaron y corrigieron esos datos utilizando literatura taxonómica más reciente, así como correcciones provistas por especialistas. Se identificaron las especies y géneros endémicos por familia, así como los géneros que contienen más de 10 especies endémicas. Anteriormente se había hecho un primer intento por identificar las especies introducidas a La Hispaniola. Aquí se utilizaron datos publicados por Peck (2005) sobre los escarabajos introducidos a Cuba, para suplementar esa información. Además, se comparó la diversidad de especies en La Hispaniola con la reportada para otras islas del Caribe y se examinó la relación especies - área para las Antillas Mayores mediante un análisis de regresión lineal. Se incluyeron datos de algunos inventarios faunísticos que aún se encuentran en preparación (Stewart B. Peck, com. pers., 2010). Finalmente, para examinar las tendencias históricas de la taxonomía sobre los coleópteros en La Hispaniola, se analizó el patrón de descripción de nuevas especies endémicas por décadas, desde los inicios de la taxonomía zoológica hasta el presente.

\section{RESULTADOS Y DISCUSIÓN}

Diversidad de escarabajos. En Perez-Gelabert (2008) se había reportado que el orden Coleoptera representa cerca de un tercio del total de insectos y alrededor de un cuarto de los artrópodos conocidos para La Hispaniola. Actualmente, la diversidad conocida de escarabajos en La Hispaniola consiste en 1,973 especies, incluidas en 788 géneros y 75 familias (Tabla 1). Las siete familias más diversas y con más de 100 especies conocidas en la fauna actual son: Curculionidae (253), Chrysomelidae (211), Staphylinidae (206), Carabidae (173), Cerambycidae (148), Scarabaeidae (131) y Tenebrionidae (104). Peck (2005) reporta que Cuba comparte el 56\% de sus escarabajos nativos con La Hispaniola. Varias otras familias de escarabajos que muy probablemente estén presentes en La Hispaniola, por ser conocidas de Cuba, son: Ptiliidae, Ochodeidae, Scirtidae, Ptilodactylidae, Nosodendridae, Biphyllidae, Discolomatidae, Melandryidae, Colydiidae y Belidae. Este inventario general de escarabajos claramente indica que el conocimiento de esta fauna en La Hispaniola es todavía incipiente. Un total de 48 de las 75 familias reportadas son conocidas de 10 especies o menos, mientras que 7 familias son conocidas de una sola especie. Hasta ahora, nunca se ha realizado un inventario taxonómico comprensivo de los escarabajos en ninguna de las áreas protegidas de República Dominicana o Haití, que son los sitios identificados como ricos en biodiversidad. Para ser comprensivo, tal inventario necesitaría envolver muestreos que utilicen una amplia variedad de técnicas de colecta por varios años y de esta forma tomen en cuenta la variabilidad temporal que se observa regularmente en los ecosistemas de la isla. Recientemente, se completó el primer trabajo local de tesis en Licenciatura en Biología en el cual se logra un estudio sobre la ecología y distribución de la fauna de escarabajos de un área determinada de República Dominicana (De la Rosa, 2009). 
Endemismo. El endemismo varía aún dentro de las familias y parece estar directamente relacionado con la capacidad de dispersión de las especies. En términos generales, más de la mitad de los escarabajos reportados son exclusivos de La Hispaniola (1,045 especies, 53.0\%). Esto significa que más de la mitad de las especies han evolucionado en la isla. De las 75 familias reportadas, 33 presentan un endemismo superior al 50\% (Tabla 1). El endemismo a nivel de género envuelve linajes aún más antiguos, los que probablemente también evolucionaron en la isla o allí encontraron refugio quedando como relictos. Un total de 28 géneros parecen ser endémicos de La Hispaniola (Tabla 2). Entre estos predominan las familias Cerambycidae y Chrysomelidae, que son escarabajos fitófagos. También se encuentran tres géneros de Elmidae, dos de los cuales habitan exclusivamente aguas subterráneas. Peck (2005) señala que las Indias Occidentales contienen en total más de 190 géneros endémicos de escarabajos.

Unos 20 géneros contienen más de 10 especies endémicas de La Hispaniola, indicando que estos linajes han experimentado significativa radiación evolutiva en la isla y se han diversificado hasta producir numerosas especies (Tabla 3). Estos corresponden, principalmente, a las familias Scarabaeidae, Lampyridae, Cantharidae y Chrysomelidae. El género Phyllophaga (Scarabaeidae), revisado recientemente en La Hispaniola por Woodruff \& Sanderson (2004), es el de mayor número de especies endémicas (48). Las familias con porcentajes más altos de endemismo son Cantharidae (98.1\%), Lampyridae (97.0\%), Melyridae (93.4\%), Anthribidae (91.8\%), Erotylidae (78.9\%), Chrysomelidae (77.7\%) y Scarabaeidae (68.7\%). Un alto porcentaje de los escarabajos por descubrir en La Hispaniola deberán también ser especies endémicas. No sería sorprendente que el porcentaje general de endemismo pudiera alcanzar hasta más del $60 \%$.

Fósiles en ámbar. La República Dominicana posee depósitos fosilíferos de ámbar que datan 15-20 millones de años (época Mioceno). Por su pequeño tamaño y frecuente asociación con los troncos de árboles, los insectos eran uno de los grupos que con mayor frecuencia quedaban atrapados y eran preservados en la resina que produjo este ámbar. Actualmente se han descrito más de 600 especies de insectos del ámbar dominicano, entre los que se incluyen 184 especies de escarabajos fósiles pertenecientes a 29 familias (Tabla 1). La caracterización taxonómica de esta abundante fauna fósil es también preliminar, pero en general los fósiles demuestran que hace 15-20 millones de años la isla ya contenía una fauna de artrópodos y vertebrados igual o más diversa que la del presente (Perez-Gelabert, 1999). Cuatro familias de escarabajos (Lucanidae, Micromalthidae, Mycetophagidae y Scraptiidae) son todavía conocidas sólo de especies fósiles en ámbar, aunque se espera que por lo menos algunas de ellas se encuentren en la fauna actual. En el ámbar dominicano, la mayoría de escarabajos fósiles pertenecen a géneros actuales, aunque también se encuentran 20 genéros aparentemente ya extintos. Debido a que la diversificación de los escarabajos ocurrió hacia finales del Cretácico y principios del Terciario, los fósiles del ámbar dominicano son muy recientes para aportar información sobre esos eventos. Pero estos fósiles si proveen datos que pueden servir para documentar el origen, extinción y patrones biogeográficos de grupos específicos en la región neotropical. Entre los escarabajos fósiles reportados, la mayoría de familias son conocidas de unas pocas especies (16 familias por una sola especie). Las familias con mayor número de especies fósiles descritas son Curculionidae (49), Tenebrionidae (28) y Staphylinidae (24).

Escarabajos introducidos. Las especies introducidas desde otras áreas geográficas pueden, por tal razón, carecer de enemigos naturales que las mantengan bajo control. Estas, a menudo, resultan ser dañinas y agresivas en su nuevo ambiente, pudiendo desplazar a especies nativas por competencia o causar daños a la agricultura. Las introducciones de insectos ocurren mayormente de manera accidental, a través de las importaciones de alimentos, madera y otros productos. Otras veces, especialistas en control biológico realizan introducciones intencionales, con la finalidad de proveer enemigos naturales que limiten la expansión de especies plagas. En total, se encontraron 56 especies de escarabajos introducidas en La Hispaniola (Tabla 4). La mayoría son plagas menores de plantas cultivadas y productos agrícolas que han sido introducidas desde 
diferentes partes del mundo a través del comercio. Las dos especies de mariquitas, Rodolia cardinalis y Cryptolaemus montrouzieri (Coccinellidae), fueron introducidas intencionalmente durante los años 1930's con la finalidad de combatir las plagas de escamas o Coccoidea (GómezMenor, 1941). Otras especies de coccinélidos, también introducidas en ese tiempo, fueron Pentilia castanea y Cryptognatha nodiceps, pero por no encontrarlas ahora parecería que no se establecieron. Más recientemente, los curculiónidos Elaeidobius subvittatus (Faust, 1898) y Elaeidobius kamerunicus (Faust, 1898) fueron introducidos a República Dominicana para ayudar en la polinización de la palma aceitera (A.Abud, com. pers., 2011). Una de las especies introducidas de escarabajos con mayor impacto económico en La Hispaniola es la broca del café (Hypothenemus hampei), detectada en la isla por primera vez en 1995 (ver Contreras y Camilo, 2007).

Comparación con otras islas. A modo comparativo, la Tabla 5 muestra los números de especies de escarabajos actuales reportados de La Hispaniola y otras islas de las Indias Occidentales y Tobago. Como lo predice la teoría de biogeografía de islas, la diversidad en las islas antillanas está relacionada con el tamaño de los territorios insulares y su distancia de los continentes adyacentes. A pesar de tratarse de datos preliminares, puede observarse que Cuba y La Hispaniola tienen faunas significativamente más grandes que Jamaica y Puerto Rico. Pero debido a que los insectos de La Hispaniola todavía no han sido comprensivamente muestreados ni estudiados, se espera que la riqueza de escarabajos en La Hispaniola sea aún más cercana a la de Cuba. Un análisis de regresión lineal de la fauna de escarabajos en las cuatro Antillas Mayores (fig. 1) muestra una relación de especies - área estadísticamente significativa. La relación predice que el número de especies de escarabajos en La Hispaniola debe ser mucho mayor, claramente sobrepasando las 2,000 especies.

Las islas de las Antillas Menores, de tamaños más reducidos y con características ecológicas menos complejas, tienen en general faunas más limitadas. Pero es necesario destacar el número relativamente alto de familias (107) y también especies encontradas en el archipiélago de Guadalupe. Sería lógico pensar que con inventarios más detallados la mayoría de esas familias deberán también encontrarse en las Antillas Mayores. Por otro lado, la pequeña isla de Tobago muestra un significativo incremento, reflejando su cercanía a América del Sur. Sin embargo, las dos islas más pequeñas, Montserrat y Guana, ambas objeto de inventarios recientes, ejemplifican lo que podría ser la capacidad de especies de escarabajos en islas tropicales. Como ejemplo extremo, Guana, con sus bosques originales relativamente intactos y objeto de un inventario de múltiples años en un terreno relativamente fácil de muestrear, ostenta 411 especies de escarabajos en sólo $3.4 \mathrm{~km}^{2}$ de área.

Coleopterofauna total. Es dificil precisar el número de escarabajos que aún quedan por descubrir en La Hispaniola. Muchas familias de escarabajos parecen contener el doble o varias veces el número de las especies ya reportadas. Un ejemplo reciente es la revisión taxonómica de las luciérnagas (Lampyridae), a partir de especímenes exclusivamente provenientes de la República Dominicana, realizada por Kazantsev y Perez-Gelabert (2009). En ese trabajo se describieron 33 especies nuevas, duplicando a 66 (64 endémicas) el total conocido para la isla. Para Haití sólo se han reportado 14 especies y sólo una se conoce exclusivamente de este país. A nivel de géneros, un ejemplo es la reciente revisión del género antillano de Tenebrionidae Nesocyrtosoma (Hopp \& Ivie, 2009). Este género se conocía en La Hispaniola de dos especies actuales y cinco fósiles (una originalmente descrita en otro género). La revisión agregó 19 nuevas especies actuales y 1 fósil, multiplicando así varias veces el número de especies conocidas para este género en la isla.

Si intentáramos estimar el total de escarabajos que habitan La Hispaniola, podría ser útil extrapolar la proporción de este grupo que incluiría el total de insectos estimados para la isla. De manera conservadora, podríamos estimar un total de alrededor de 10,000 especies de insectos. En Perez-Gelabert (2008) se encontró que los escarabajos representaban casi el 32\% del total 
de insectos reportados hasta entonces. Este estimado es algo más bajo que el presunto $36-$ $40 \%$ del total de insectos en el mundo. La diferencia podría estar relacionada con el relativo bajo conocimiento de los escarabajos en la isla. En todo caso, el $32-40 \%$ del total arrojaría la cifra de 3,200 - 4,000 especies de escarabajos. Por supuesto que, si 10,000 especies de insectos resultara ser un estimado muy bajo, el número de escarabajos en la isla podría sobrepasar las 5,000 especies.

Tendencias históricas. Wetherbee (1985) produjo lo que probablemente es el único intento hasta ahora de reconstruir aspectos sobre la historia de las exploraciones coleopterológicas en La Hispaniola. En su trabajo identifica al crisomélido Leucocera quinquepunctata (Linnaeus, 1767) como la primera especie endémica en ser descrita de la isla y da una lista de los escarabajos endémicos conocidos hasta 1971, incluyendo entre otros detalles el colector y la localidad tipo. La figura 2 examina las tendencias históricas de la taxonomía de escarabajos en la isla. Se grafica el número de especies endémicas de escarabajos descritas para La Hispaniola por décadas, desde inicios de la taxonomía zoológica hasta el presente. Por cerca de 200 años fue relativamente poco lo que se estudió sobre escarabajos autóctonos de la isla. Hasta 1920 se habían descrito sólo 193 especies endémicas. En la gráfica se observa un primer pico de 130 especies en la década de 1931-1940, que resulta principalmente de los carábidos descubiertos a partir de las expediciones a Haití y República Dominicana de Philip J. Darlington, Jr. (Museo de Zoología Comparada, Universidad de Harvard) durante los 1920's y 1930's, junto a las nuevas especies de bupréstidos y cerambícidos descritas por W. S. Fisher (United States Department of Agriculture) y de crisomélidos por Doris H. Blake (United States National Museum of Natural History, Smithsonian Institution). El segundo y más pronunciado pico, con 201 especies endémicas descritas, ocurre en la década de 2001-2010. Este marcado incremento en el número de nuevas especies refleja el renovado interés de la comunidad taxonómica internacional por el estudio de los escarabajos de La Hispaniola y el Caribe, así como la mayor facilidad para lograr trabajos de campo, principalmente en la República Dominicana.

Conservación. La pérdida de hábitats es la peor amenaza a las poblaciones de escarabajos y otros artrópodos. El alto número de géneros endémicos de La Hispaniola y la región del Caribe pone en evidencia la excepcional concentración de especies únicas del área, al tiempo que provee soporte a la designación de la región como un "hotspot" de biodiversidad, ameritando mayores esfuerzos para su conservación y estudio. Sin embargo, poco sabemos sobre el estado de conservación de las poblaciones de escarabajos y otros insectos en la isla. Hasta ahora no tenemos datos concretos para señalar que ninguna especie se encuentre en peligro de extinción. Sí es cierto que muchas especies de insectos caribeños tienen poblaciones pequeñas y viven restringidas a hábitats particulares. Estas características las hacen más vulnerables a ser extinguidas, al menos localmente, cuando por ejemplo se malogra un bosque de montaña en un área con reductos ecológicamente únicos como los que se encuentran en la Sierra de Neiba o la Sierra de Bahoruco. Dada la degradación física de muchos ambientes en múltiples lugares de República Dominicana y Haití, es urgente que se adopten medidas que frenen este deterioro. Objetivos primarios deberían ser la recuperación de bosques y cuencas de ríos, así como la reducción de la contaminación química resultante del uso excesivo de plaguicidas. El manejo adecuado de la basura de las ciudades y pueblos frenaría mucho la contaminación orgánica y la proliferación de plagas asociadas. También sería recomendable, en la mayor medida posible, la eliminación de especies exóticas dañinas, así como redoblar los esfuerzos por impedir nuevas introducciones.

\section{CONCLUSIONES}

Por ser mucho más diversa de lo ya reportado, la fauna de escarabajos en La Hispaniola es merecedora de mayor atención por parte de los investigadores y de mayor protección por las instituciones incumbentes y la población en general. La mayoría de estas especies han 
evolucionado luego de miles de años en la isla y no se encuentran en ningún otro lugar del mundo. La disponibilidad de ancestros fósiles en ámbar presenta interesantes oportunidades para el estudio de la evolución de esta fauna. Es probable que, con la destrucción y transformación de muchos hábitats en La Hispaniola, múltiples especies de escarabajos se hayan extinguido sin apenas darnos cuenta. Sabemos que faltan por lo menos la mitad de las especies por ser descritas y reportadas, por lo que su caracterización taxonómica debería ser una tarea prioritaria a realizarse de manera intensiva. Los investigadores locales estarían en gran ventaja para contribuir a este inventario y también a la caracterización biológica de las especies.

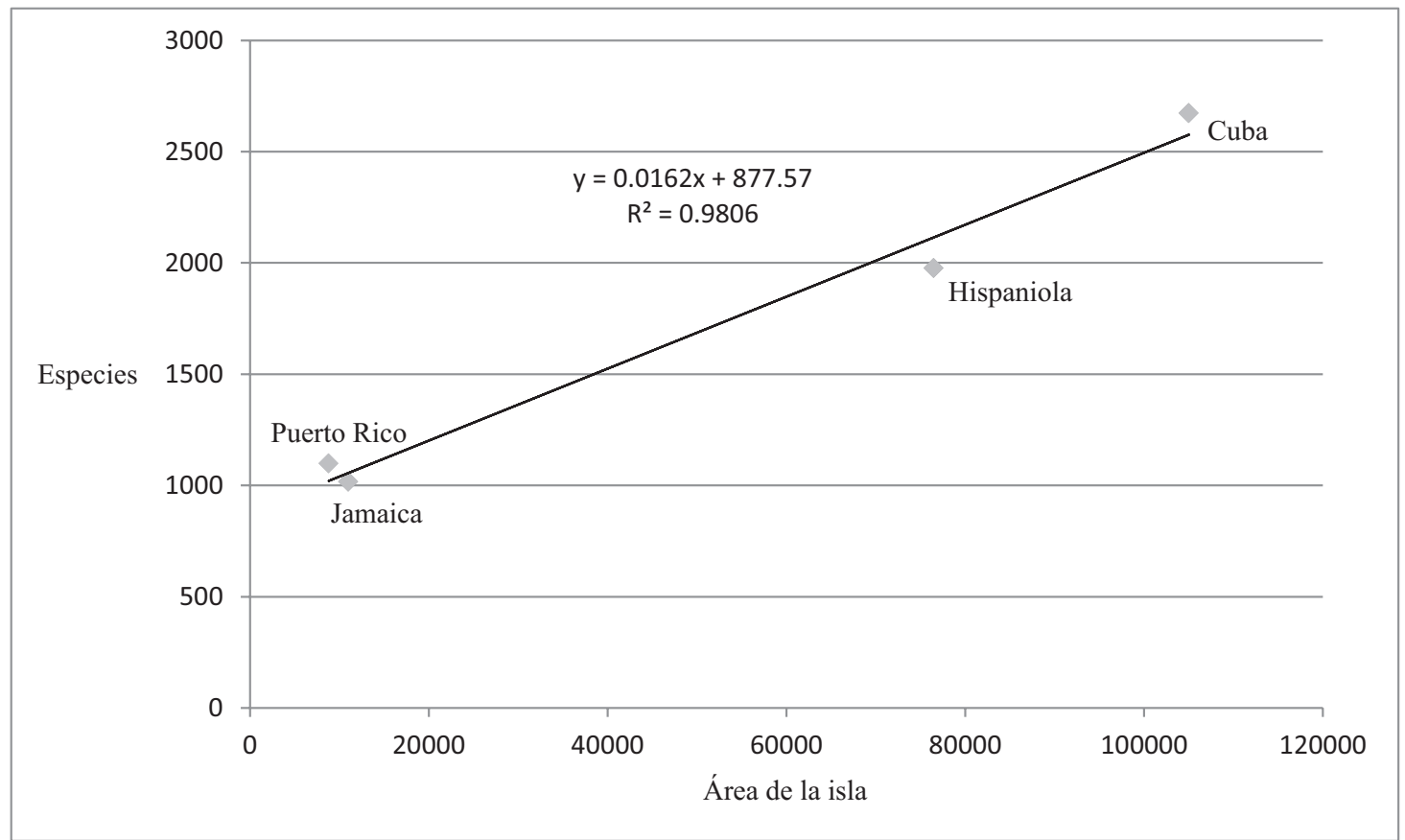

Figura 1. Análisis de regresión lineal de la fauna de escarabajos en las Antillas Mayores. La relación de especies - área es altamente significativa.

Tabla 1. Diversidad de escarabajos en La Hispaniola por familias.*

\begin{tabular}{|lccccc|}
\hline \multicolumn{1}{c}{ Familias } & Géneros & Especies & Especies endémicas & $\begin{array}{c}\text { Fósiles } \\
\text { en ámbar }\end{array}$ & $\begin{array}{c}\text { Especies } \\
\text { introducidas }\end{array}$ \\
\hline Anobiidae & 5 & 8 & $1(12.5 \%)$ & 0 & 0 \\
Anthicidae & 9 & 20 & $4(20.0 \%)$ & 0 & 3 \\
Anthribidae & 14 & 73 & $67(91.8 \%)$ & 0 & 1 \\
Apionidae & 1 & 12 & $8(66.7 \%)$ & 1 & 0 \\
Attelabidae & 3 & 17 & $7(41.2 \%)$ & 0 & 0 \\
Bostrichidae & 10 & 13 & $0(0.0 \%)$ & 0 & 2 \\
Bothrideridae & 1 & 2 & $0(0.0 \%)$ & $1(100.0 \%)$ & 1 \\
Brachypsectridae & 1 & 1 & & 0 & 0 \\
\hline
\end{tabular}

\footnotetext{
*Revisiones taxonómicas recientes han reducido a Scydmaenidae y Languriidae a subfamilias de Staphylinidae y Erotylidae, respectivamente.
} 
Tabla 1 (continuación).

\begin{tabular}{|c|c|c|c|c|c|}
\hline Familias & Géneros & Especies & Especies endémicas & $\begin{array}{l}\text { Fósiles } \\
\text { en ámbar }\end{array}$ & $\begin{array}{c}\text { Especies } \\
\text { introducidas }\end{array}$ \\
\hline Brentidae & 13 & 23 & $1(4.3 \%)$ & 0 & 1 \\
\hline Bruchidae & 12 & 32 & $0(0.0 \%)$ & 0 & 3 \\
\hline Buprestidae & 24 & 48 & $29(60.4 \%)$ & 1 & 2 \\
\hline Cantharidae & 6 & 52 & $51(98.1 \%)$ & 0 & 0 \\
\hline Carabidae & 70 & 173 & $81(46.8 \%)$ & 12 & 1 \\
\hline Cerambycidae & 72 & 148 & $91(61.5 \%)$ & 14 & 1 \\
\hline Ceratocanthidae & 1 & 1 & $1(100.0 \%)$ & 0 & 0 \\
\hline Cerylonidae & 6 & 9 & $2(22.2 \%)$ & 0 & 0 \\
\hline Chelonariidae & 1 & 1 & $0(0.0 \%)$ & 0 & 0 \\
\hline Chrysomelidae & 71 & 211 & $164(77.7 \%)$ & 15 & 0 \\
\hline Ciidae & 3 & 4 & $0(0.0 \%)$ & 0 & 0 \\
\hline Cleridae & 6 & 10 & $7(70.0 \%)$ & 1 & 1 \\
\hline Coccinellidae & 22 & 30 & $11(36.7 \%)$ & 0 & 2 \\
\hline Corylophidae & 1 & 2 & $2(100.0 \%)$ & 0 & 0 \\
\hline Cryptophagidae & 1 & 1 & $1(100.0 \%)$ & 0 & 0 \\
\hline Curculionidae & 103 & 253 & $96(37.9 \%)$ & 49 & 15 \\
\hline Dermestidae & 2 & 9 & $3(33.3 \%)$ & 5 & 3 \\
\hline Dryopidae & 1 & 1 & $0(0.0 \%)$ & 0 & 0 \\
\hline Dytiscidae & 13 & 24 & $0(0.0 \%)$ & 1 & 0 \\
\hline Elateridae & 15 & 45 & $16(35.6 \%)$ & 0 & 0 \\
\hline Elmidae & 6 & 10 & $7(70.0 \%)$ & 0 & 0 \\
\hline Endomychidae & 2 & 2 & $1(50.0 \%)$ & 1 & 0 \\
\hline Erotylidae & 5 & 19 & $15(78.9 \%)$ & 1 & 1 \\
\hline Eucnemidae & 1 & 1 & $0(0.0 \%)$ & 0 & 0 \\
\hline Geotrupidae & 2 & 6 & $4(66.7 \%)$ & 0 & 0 \\
\hline Gyrinidae & 3 & 5 & $0(0.0 \%)$ & 0 & 0 \\
\hline Haliplidae & 1 & 4 & $0(0.0 \%)$ & 0 & 0 \\
\hline Heteroceridae & 3 & 3 & $0(0.0 \%)$ & 0 & 0 \\
\hline Histeridae & 11 & 17 & $2(11.8 \%)$ & 1 & 2 \\
\hline Hybosoridae & 2 & 3 & $1(33.3 \%)$ & 3 & 1 \\
\hline Hydraenidae & 3 & 3 & $1(33.3 \%)$ & 0 & 0 \\
\hline Hydrophilidae & 11 & 21 & $0(0.0 \%)$ & 1 & 0 \\
\hline Laemophloeidae & 5 & 9 & $0(0.0 \%)$ & 0 & 0 \\
\hline Lampyridae & 10 & 66 & $64(97.0 \%)$ & 0 & 0 \\
\hline Latridiidae & 4 & 8 & $6(75.0 \%)$ & 0 & 0 \\
\hline Leiodidae & 5 & 5 & $1(20.0)$ & 1 & 0 \\
\hline Limnichidae & 4 & 5 & $0(0.0 \%)$ & 0 & 0 \\
\hline
\end{tabular}


Tabla 1 (continuación).

\begin{tabular}{|c|c|c|c|c|c|}
\hline Familias & Géneros & Especies & Especies endémicas & $\begin{array}{l}\text { Fósiles } \\
\text { en ámbar }\end{array}$ & $\begin{array}{c}\text { Especies } \\
\text { introducidas }\end{array}$ \\
\hline Lucanidae & 0 & 0 & $0(0.0 \%)$ & 1 & 0 \\
\hline Lutrochidae & 1 & 1 & $0(0.0 \%)$ & 0 & 0 \\
\hline Lycidae & 2 & 7 & $5(71.4 \%)$ & 0 & 0 \\
\hline Lymexylidae & 1 & 1 & $0(0.0 \%)$ & 1 & 0 \\
\hline Meloidae & 4 & 4 & $0(0.0 \%)$ & 1 & 0 \\
\hline Melyridae & 6 & 16 & $15(93.4 \%)$ & 0 & 0 \\
\hline Micromalthidae & 0 & 0 & $0(0.0 \%)$ & 1 & 0 \\
\hline Micropeplidae & 1 & 1 & $1(100.0 \%)$ & 0 & 0 \\
\hline Monotomidae & 1 & 1 & $0(0.0 \%)$ & 0 & 0 \\
\hline Mordellidae & 4 & 6 & $4(66.6 \%)$ & 0 & 0 \\
\hline Mycetophagidae & 0 & 0 & $0(0.0 \%)$ & 1 & 0 \\
\hline Mycteridae & 2 & 3 & $3(100.0 \%)$ & 0 & 0 \\
\hline Nitidulidae & 7 & 8 & $2(25.0 \%)$ & 2 & 0 \\
\hline Noteridae & 5 & 6 & $0(0.0 \%)$ & 0 & 0 \\
\hline Oedemeridae & 5 & 16 & $2(12.5 \%)$ & 0 & 0 \\
\hline Passalidae & 3 & 4 & $3(75.0 \%)$ & 0 & 0 \\
\hline Passandridae & 1 & 2 & $1(50.0 \%)$ & 0 & 0 \\
\hline Phalacridae & 3 & 3 & $3(100.0 \%)$ & 0 & 0 \\
\hline Phengodidae & 1 & 1 & $1(100.0 \%)$ & 0 & 0 \\
\hline Psephenidae & 2 & 2 & $2(100.0 \%)$ & 0 & 0 \\
\hline Rhysodidae & 2 & 5 & $5(100.0 \%)$ & 3 & 0 \\
\hline Ripiphoridae & 1 & 1 & $0(0.0 \%)$ & 0 & 0 \\
\hline Salpingidae & 2 & 2 & $2(100.0 \%)$ & 0 & 0 \\
\hline Scarabaeidae & 31 & 131 & $90(68.7 \%)$ & 4 & 4 \\
\hline Scraptiidae & 0 & 0 & $0(0.0 \%)$ & 1 & 0 \\
\hline Silphidae & 1 & 1 & $1(100.0 \%)$ & 0 & 0 \\
\hline Silvanidae & 8 & 13 & $6(46.2 \%)$ & 3 & 4 \\
\hline Smicripidae & 1 & 1 & $0(0.0 \%)$ & 0 & 0 \\
\hline Sphindidae & 1 & 1 & $1(100.0 \%)$ & 0 & 0 \\
\hline Staphylinidae & 84 & 206 & $96(46.6 \%)$ & 24 & 4 \\
\hline Tenebrionidae & 36 & 104 & $54(51.9 \%)$ & 28 & 4 \\
\hline Trogidae & 2 & 2 & $0(0.0 \%)$ & 0 & 0 \\
\hline Trogossitidae & 3 & 3 & $0(0.0 \%)$ & 0 & 0 \\
\hline Zopheridae & 5 & 11 & $4(36.4 \%)$ & 7 & 0 \\
\hline 75 & 788 & 1,973 & $1,045(53.0 \%)$ & 184 & 56 \\
\hline
\end{tabular}


Tabla 2. Géneros endémicos de escarabajos en La Hispaniola.*

\begin{tabular}{|c|c|}
\hline Staphylinidae & $\begin{array}{l}\text { Hispanisella Park, } 1976 \\
\text { Sandersonella Park, } 1976\end{array}$ \\
\hline Scarabaeidae & $\begin{array}{l}\text { Endroedianibe Chalumeau, } 1981 \\
\text { Hispanioryctes Howden, } 1978\end{array}$ \\
\hline Elmidae & $\begin{array}{l}\text { Hispaniolara Brown, } 1981 \\
\text { Anommatelmis Spangler, } 1981 \\
\text { Lemalelmis Spangler, } 1981\end{array}$ \\
\hline Phengodidae & Acladocera Wittmer, 1981 \\
\hline Lampyridae & $\begin{array}{l}\text { Callopisma Motschulsky, 1853** } \\
\text { Erythrolychnia Motschulsky, } 1853\end{array}$ \\
\hline Cantharidae & Silidiscodon Leng \& Mutchler, 1922 \\
\hline Cleridae & Decorosa Opitz, 2008 \\
\hline Melyridae & Opsablechrus Wittmer, 1967 \\
\hline Tenebrionidae & $\begin{array}{l}\text { Cenophorus Mulsant \& Rey, } 1859 \\
\text { Sphaerognathium Dajoz, } 1972\end{array}$ \\
\hline Cerambycidae & $\begin{array}{l}\text { Parandraceps Giesbert, } 1998 \\
\text { Plectrocerum Dejean, } 1835 \\
\text { Hormathus Gahan, } 1890 \\
\text { Phoenicus Lacordaire, } 1869 \\
\text { Derancistrodes Galileo \& Martins, } 1993\end{array}$ \\
\hline Chrysomelidae & $\begin{array}{l}\text { Kiskeya Konstantinov \& Chamorro-Lacayo, } 2006 \\
\text { Hirtasphaera } \text { Medvedev, } 2004 \\
\text { Agathispa } \text { Weise, } 1905 \\
\text { Asteriza } \text { Chevrolat, } 1837 \\
\text { Omoteina Chevrolat, } 1837 \\
\text { Gonaives Clark, } 1987\end{array}$ \\
\hline Curculionidae & $\begin{array}{l}\text { Hormotrophus Schönherr, } 1843 \\
\text { Paululusus Howden, } 1970\end{array}$ \\
\hline
\end{tabular}

\footnotetext{
* Un total de 28 géneros clasificados en 12 familias.

**Callopisma es también citado de Cuba, aunque de acuerdo a Kazantsev \& Perez-Gelabert (2009) y S. V. Kazantsev (com. pers., 2010) esas especies probablemente deban transferirse a otro género.
} 
Tabla 3. Géneros con 10 o más especies endémicas en La Hispaniola.

\begin{tabular}{|c|c|c|}
\hline Familias & Géneros & No. especies endémicas \\
\hline Carabidae & Platynus Bonelli, 1810 & 26 \\
\hline Staphylinidae & Euconnus Thomson, 1862 & 28 \\
\hline Scarabaeidae & Phyllophaga Harris, 1826 & 48 \\
\hline \multirow[t]{4}{*}{ Lampyridae } & Erythrolychnia Motschulsky, 1853 & 12 \\
\hline & Lychnacris Motschulsky, 1853 & 13 \\
\hline & Heterophotinus E. Olivier, 1894 & 10 \\
\hline & Robopus Motschulsky, 1853 & 10 \\
\hline \multirow[t]{2}{*}{ Cantharidae } & Silis Charpentier, 1825 & 16 \\
\hline & Tytthonyx LeConte, 1851 & 19 \\
\hline Erotylidae & Notaepytus Skelley, 2009 & 11 \\
\hline Tenebrionidae & Nesocyrtosoma Marcuzzi, 1976 & 20 \\
\hline Cerambycidae & Leptostylopsis Dillon, 1956 & 13 \\
\hline \multirow[t]{4}{*}{ Chrysomelidae } & Aedmon Clark, 1860 & 18 \\
\hline & Cryptocephalus Geoffroy, 1762 & 13 \\
\hline & Chalcosicya Blake, 1930 & 15 \\
\hline & Metachroma Chevrolat, 1837 & 15 \\
\hline \multirow[t]{2}{*}{ Anthribidae } & Homocloeus Jordan, 1904 & 13 \\
\hline & Ormiscus Waterhouse, 1845 & 33 \\
\hline \multirow[t]{2}{*}{ Curculionidae } & Exophthalmus Schönherr, 1823 & 14 \\
\hline & Lachnopus Schönherr, 1840 & 23 \\
\hline
\end{tabular}

Tabla 4. Especies de escarabajos introducidas en La Hispaniola.*

\begin{tabular}{|c|c|}
\hline Carabidae & Buprestidae \\
\hline $\begin{array}{l}\text { Perigona nigriceps (Dejean, 1831) } \\
\text { Histeridae }\end{array}$ & $\begin{array}{l}\text { Aphanisticus cochinchinae seminulum } \\
\text { Obenberger, } 1929 \\
\text { Melanophila acuminata (De Geer, 1774) }\end{array}$ \\
\hline Carcinops troglodytes (Paykull, 1811) & Dermestidae \\
\hline Staphylinidae & $\begin{array}{l}\text { Trogoderma anthrenoides (Sharp, 1902) } \\
\text { Trogoderma bicinctum Reitter, } 1881\end{array}$ \\
\hline $\begin{array}{l}\text { Anotylus glareosus (Wollaston, 1854) } \\
\text { Oxytelus incisus Motschulsky, 1857 } \\
\text { Philonthus discoideus (Gravenhorst, 1802) }\end{array}$ & Trogoderma granarium Everts, 1898 \\
\hline $\begin{array}{l}\text { Philonthus ventralis (Gravenhorst, 1802) } \\
\text { Hybosoridae }\end{array}$ & $\begin{array}{l}\text { Apate monachus Fabricius, } 1775 \\
\text { Dinoderus minutus (Fabricius, 1775) }\end{array}$ \\
\hline Hybosorus illigeri Reiche, 1853 & Cleridae \\
\hline Scarabaeidae & $\begin{array}{l}\text { Necrobia rufipes (De Geer, 1775) } \\
\text { Silvanidae }\end{array}$ \\
\hline $\begin{array}{l}\text { Cyclocephala notata (Illiger, 1806) } \\
\text { Digitonthophagus gazella (Fabricius, 1787) } \\
\text { Labarrus lividus (Olivier, 1789) } \\
\text { Nialaphodius nigrita (Fabricius, 1801) }\end{array}$ & $\begin{array}{l}\text { Ahasverus advena (Waltl, 1832) } \\
\text { Monanus concinnulus (Walker, 1858) } \\
\text { Oryzaephilus surinamensis (Linnaeus, 1758) } \\
\text { Silvanoprus scuticollis (Walker, 1859) }\end{array}$ \\
\hline
\end{tabular}

\footnotetext{
*Un total de 56 especies en 19 familias.
} 
Tabla 4 (continuación).

\begin{tabular}{|c|c|}
\hline Erotylidae & Anthribidae \\
\hline Aegithus clavicornis (Linnaeus, 1758) & Araecerus fasciculatus (De Geer, 1775) \\
\hline Coccinellidae & Brentidae \\
\hline Cryptolaemus montrouzieri Mulsant, 1853 & Cylas formicarius (Fabricius, 1798) \\
\hline Rodolia cardinalis (Mulsant, 1850) & Curculionidae \\
\hline Tenebrionidae & Caulophilus orizae (Gyllenhal, 1838) \\
\hline Epitragus aurulentus Kirsch, 1866 & Conotrachelus sapotae Barber, 1923 \\
\hline Tribolium castaneum (Herbst, 1797) & Elaeidobius kamerunicus (Faust, 1898) \\
\hline Tribolium confusum Jacquelin du Val, 1868 & Elaeidobius subvitatus (Faust, 1898) \\
\hline Ulomoides ocularis (Casey, 1891) & Euscepes postfasciatus (Fairmaire, 1849) \\
\hline Anthicidae & Rhynchophorus palmarum (Linnaeus, 1764) \\
\hline & Sitophilus granarius (Linnaeus, 1758) \\
\hline Omonadus floralis (Linnaeus, 1758) & Sitophilus oryzae (Linnaeus, 1763) \\
\hline Sticticomus tobias (Marseul, 1879) & Sitophilus zeamays Motschulsky, 1855 \\
\hline Vacusus vicinus (Laferté-Sénectère, 1849) & Coccotrypes advena Blandford, 1894 \\
\hline Cerambycidae & Coccotrypes carpophagus (Hornung, 1842) \\
\hline Isotomus comptus (Mannerheim, 1825) & Hypothenemus crudiae (Panzer, 1791) \\
\hline & Hypothenemus hampei (Ferrari, 1867) \\
\hline Bruchidae & Hypothenemus javanus (Eggers, 1908) \\
\hline Acanthoscelides obtectus (Say, 1831) & Hypothenemus setosus (Eichhoff, 1868) \\
\hline Bruchus pisorum (Linnaeus, 1758) & Pagiocerus frontalis (Fabricius, 1801) \\
\hline Zabrotes subfasciatus (Boheman, 1833) & Scolytogenes knabi (Hopkins, 1915) \\
\hline
\end{tabular}

Tabla 5. Números de especies de escarabajos en islas de las Indias Occidentales y Tobago.*

\begin{tabular}{|c|c|c|c|c|c|}
\hline Isla & Área $\left(\mathrm{km}^{2}\right)$ & Especies & Géneros & Familias & Referencia \\
\hline Cuba & 105,006 & 2,673 & 954 & 87 & Peck (2005) \\
\hline La Hispaniola & 76,480 & 1,973 & 788 & 75 & Este trabajo \\
\hline Jamaica & 10,991 & 1,017 & 501 & 49 & Perez-Gelabert (en prep.) \\
\hline Puerto Rico & 8,800 & 1,098 & $? ?$ & 58 & Maldonado Capriles (1996) \\
\hline Guana & 3.4 & 411 & 240 & 57 & Valentine \& Ivie en Lazell (2005) \\
\hline Bahamas & 13,940 & 996 & 552 & 74 & Turnbow \& Thomas (2008) \\
\hline Montserrat & 102 & 718 & $? ?$ & 63 & Ivie et al. (2008) \\
\hline Martinica & 1,182 & 258 & 188 & 40 & Peck (en prep.) \\
\hline $\begin{array}{l}\text { Northern Leeward } \\
\text { Islands** }\end{array}$ & 849.5 & 218 & 155 & 26 & Peck (2011) \\
\hline $\begin{array}{l}\text { Guadalupe } \\
\text { Archipielagos }\end{array}$ & 1,628 & 1,325 & 699 & 107 & Peck (en prep.) \\
\hline Santa Lucia & 616 & 175 & 135 & 25 & Peck (2009b) \\
\hline San Vicente & 344 & 536 & 371 & 62 & Peck (2010) \\
\hline Dominica & 751 & 347 & 269 & 42 & Peck (2006) \\
\hline Granada & 344 & 507 & 345 & 51 & Woodruff et al. (1998) \\
\hline Barbados & 445 & 254 & 202 & 40 & Peck (2009a) \\
\hline Tobago & 300 & 672 & $? ?$ & 69 & Peck, Cook \& Hardy (2002) \\
\hline
\end{tabular}

\footnotetext{
*Números derivados de trabajos en preparación son preliminares. \$Familias contadas a partir de los trabajos de Wolcott (1936, 1941); **Anguilla (26 km2), Antigua (281 km2), Barbuda (160.5 km2), Nevis (93 km2), Saba (13 km2), St. Eustatius (21 km2), St. Kitts (168 km2) y St. Martin-Sint Marteen (87 km2). §Grande Terre, Base Terre, la Désirade, Marie-Galante y les Saintes.
} 


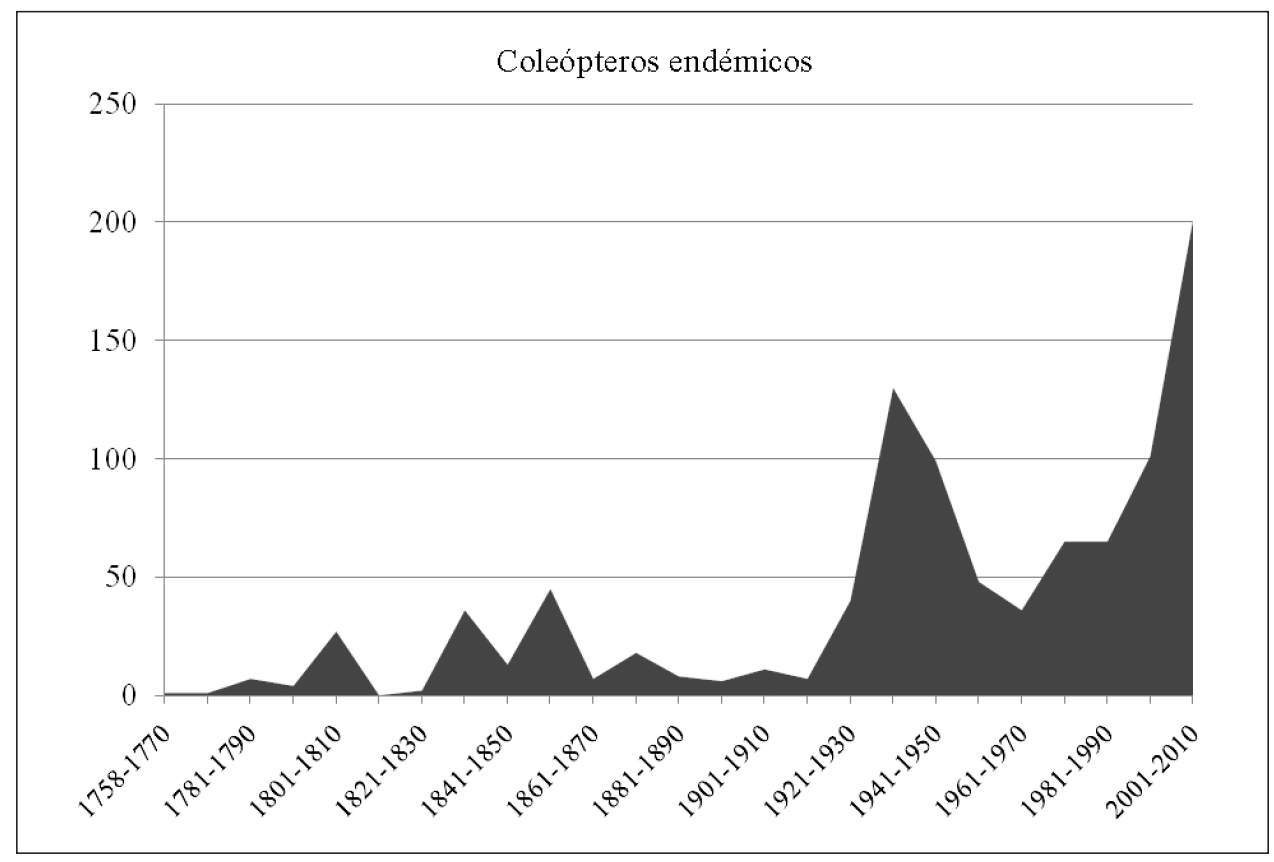

Figura 2. Número de especies endémicas de escarabajos descritas de La Hispaniola por décadas, desde inicios de la taxonomía zoológica (1758) hasta el presente.

\section{AGRADECIMIENTOS}

Stewart B. Peck (Carleton University, Ottawa, Canada) hizo comentarios y sugerencias, ofreciendo múltiples datos que sirvieron para mejorar y enriquecer este trabajo. Julio A. Genaro (York University, Toronto, Canada) leyó una version preliminar del manuscrito haciendo correcciones y comentarios de utilidad. Sergey V. Kazantsev (Insect Centre, Moscú, Rusia) ayudó a esclarecer mis ideas sobre Lampyridae. Alfred F. Newton (Field Museum of Natural History, Chicago, IL) contribuyó con importantes correcciones a mi lista de escarabajos publicada en el 2008. Abraham Abud (Santo Domingo) aportó datos sobre especies introducidas.

\section{LITERATURA CITADA}

Chapman, A. D. 2009. Numbers of Living Species in Australia and the World. 2nd Edition. Report for the Australian Biological Resources Study, Canberra, Australia, 78 pp.

Contreras, T. y J. E., Camilo. 2007. Situación de la broca del café en la República Dominicana. 43-55 pp. En: Barrera, J. F., A. García, V. Domínguez \& C. Luna (eds.), La Broca del Café en América Tropical: Hallazgos y Enfoques. Sociedad Mexicana de Entomología y Colegio de la Frontera Sur, México.

De la Rosa F., V. I. 2009. Ecología y Distribución de Coleópteros en El Matadero, Prov. Peravia, República Dominicana. Tesis de Licenciatura en Biología, Universidad Autónoma de Santo Domingo, República Dominicana. 38 p. [no publicado].

Gómez-Menor, J. 1941. Cóccidos de la República Dominicana (Hem. Cocc.). EOS, 16: 125-143.

Hedges, S. B. 1996. Historical biogeography of West Indian vertebrates. Annual Review of Ecology and Systematics, 27: 163-196. 
Hopp, K. J. y M. A. Ivie. 2009. A revision of the West Indian genus Nesocyrtosoma Marcuzzi (Coleoptera: Tenebrionidae). The Coleopterists' Society Monographs, Supplement to The Coleopterists' Bulletin, 63 (4): 1-138.

Ivie, M. A., K. A. Marske, I. A. Foley, K. A. Guerrero, y L. L. Ivie. 2008. Invertebrates of the Centre Hills and Montserrat, with emphasis on beetles. 56-89 pp. In: Young, R. P. (ed.), A biodiversity assessment of the Centre Hills, Montserrat. Durrell Conservation Monograph No. 1. Durrell Wildlife Conservation Trust, Jersey, Channel Islands.

Kazantsev, S. V. y D. E. Perez-Gelabert. 2008. Fireflies of Hispaniola (Coleoptera: Lampyridae). Russian Entomological Journal, 17: 367-402.

Lazell, J. 2005. Island: Fact and Theory in Nature. University of California Press, Berkeley, $382 \mathrm{pp}$.

Maldonado Capriles, J. 1996. The status of insect alpha taxonomy in Puerto Rico after the scientific survey. Annals of the New York Academy of Sciences, 776: 201-216.

Mejía, M. y R. García. 2007. La flora y vegetación de La Hispaniola. 30-33 pp. En: Fernández, E., Hispaniola: Biodiversidad a través de un recorrido fotográfico. Harvard University Press, Cambridge.

Peck, S. B. 2005. A checklist of the beetles of Cuba with data on distributions and bionomics (Insecta: Coleoptera). Arthropods of Florida and Neighboring Land Areas, 18: vi + 241 pp.

Peck, S. B. 2006. The beetle fauna of Dominica, Lesser Antilles (Insecta: Coleoptera): diversity and distribution. Insecta Mundi, 20: 165-209.

Peck, S. B. 2009a. The beetles of Barbados, West Indies (Insecta: Coleoptera): diversity, distribution and faunal structure. Insecta Mundi, 0073: 1-51.

Peck, S. B. 2009b. The beetles of St. Lucia, Lesser Antilles (Insecta: Coleoptera): diversity and distributions. Insecta Mundi, 0106: 1-34.

Peck, S. B. 2010. The beetles of the island of St. Vincent, Lesser Antilles (Insecta: Coleoptera); diversity and distributions. Insecta Mundi, 0144: 1-77.

Peck, S. B. 2011. The diversity and distributions of the beetles (Insecta: Coleoptera) of the northern Leeward Islands, Lesser Antilles (Anguilla, Antigua, Barbuda, Nevis, Saba, St. Barthélemy, St. Eustatius, St. Kitts and St. Martin-St. Maarten. Insecta Mundi, 0159: 1-54.

Peck, S. B., J. Cook y J. D. Hardy, Jr. 2002. Beetle fauna of the island of Tobago, Trinidad and Tobago, West Indies. Insecta Mundi, 16: 9-23.

Perez-Gelabert, D. E. 1999. Catálogo sistemático y bibliografía de la biota fósil del ámbar dominicano. Hispaniolana (n. s.), 1: 1-65.

Perez-Gelabert, D. E. 2008. Arthropods of Hispaniola (Dominican Republic and Haiti): A checklist and bibliography. Zootaxa, 1831: 1-530.

Pindell, J. L. y S. F. Barrett. 1990. Geological evolution of the Caribbean region: a plate-tectonic perspective. 405-432 pp. In: Dengo, G. \& J. E. Case (eds.), The Geology of North America, vol. H, The Caribbean Region, Geological Society of America, Boulder, Co. 
Rosen, D. E. 1976. A vicariance model of Caribbean biogeography. Systematic Zoology, 24: 431-464.

Turnbow, R. H., Jr. y M. C. Thomas. 2008. An annotated checklist of the Coleoptera (Insecta) of the Bahamas. Insecta Mundi, 0034: 1-64.

Wetherbee, D. K. 1985. The Two-century Search for Beetles (Coleoptera) in Hispaniola. Printed by the author. Shelburne, Massachussetts, $56 \mathrm{pp}$.

Wolcott, G. N. 1936. "Insectae Borinquenses": a revised annotated check-list of the insects of Puerto Rico. Journal of the Department of Agriculture University of Puerto Rico, 20: 1-600.

Wolcott, G. N. 1941. A supplement to "Insectae Borinquenses". Journal of the Department of Agriculture University of Puerto Rico, 25: 33-158.

Woodruff, R. E., B. M. Beck, P. E. Skelley, C. y. L. Schotman y M. C. Thomas. 1998. Checklist and Bibliography of the Insects of Grenada and The Grenadines. Memoir no. 2, Center for Systematic Entomology, Gainesville, Florida, 286 pp.

Woodruff, R. E. y M. W. Sanderson. 2004. Revision of the Phyllophaga of Hispaniola (Scarabaeidae: Melolonthinae). Insecta Mundi, 18: 1-154. 